\title{
Sequence mining and transcript profiling to explore differentially expressed genes associated with lipid biosynthesis during soybean seed development
}

Huan Chen ${ }^{1,2}$, Fa-Wei Wang ${ }^{1}$, Yuan-Yuan Dong ${ }^{1}$, Nan Wang ${ }^{1}$, Ye-Peng Sun ${ }^{1}$, Xiao-Yan Li', Liang Liu ${ }^{2}$, Xiu-Duo Fan², Hai-Long Yin ${ }^{1}$, Yuan-Yuan Jing ${ }^{1}$, Xin-Yue Zhang ${ }^{1}$, Yu-Lin Li ${ }^{1}$, Guang Chen ${ }^{2^{*}}$ and Hai-Yan Li ${ }^{1,2^{*}}$

\begin{abstract}
Background: Soybean (Glycine max L.) is one of the most important oil crops in the world. It is desirable to increase oil yields from soybean, and so this has been a major goal of oilseed engineering. However, it is still uncertain how many genes and which genes are involved in lipid biosynthesis.

Results: Here, we evaluated changes in gene expression over the course of seed development using Illumina (formerly Solexa) RNA-sequencing. Tissues at 15 days after flowering (DAF) served as the control, and a total of 11592, 16594, and 16255 differentially expressed unigenes were identified at 35, 55, and 65 DAF, respectively. Gene Ontology analyses detected 113 co-expressed unigenes associated with lipid biosynthesis. Of these, 15 showed significant changes in expression levels ( $\log _{2}$ fold values $\geq 1$ ) during seed development. Pathway analysis revealed 24 co-expressed transcripts involved in lipid biosynthesis and fatty acid biosynthesis pathways. We selected 12 differentially expressed genes and analyzed their expressions using qRT-PCR. The results were consistent with those obtained from Solexa sequencing.

Conclusion: These results provide a comprehensive molecular biology background for research on soybean seed development, particularly with respect to the process of oil accumulation. All of the genes identified in our research have significance for breeding soybeans with increased oil contents.
\end{abstract}

Keywords: Gene expression, Lipid, RNA-sequencing, Soybean, Unigene

\section{Background}

Soybean [Glycine $\max$ (L.) Merrill], one of the most important oil crops in the world that accounts for less than a third (27.7\%) of the total global vegetable oil production. At present, the main producers of soybean are the United States, Brazil, Argentina, India, and China. Although world soybean oil production has steadily increased, rising from

\footnotetext{
*Correspondence: chg61@163.com; hyli99@163.com

${ }^{1}$ Ministry of Education Engineering Research Center of Bioreactor and Pharmaceutical Development, Jilin Agricultural University, Changchun, Jilin 130118, China

${ }^{2}$ College of Life Sciences, Jilin Agricultural University, Changchun, Jilin 130118, China
}

\section{Biomed Central

(c) 2012 Chen et al.; licensee BioMed Central Ltd. This is an Open Access article distributed under the terms of the Creative Commons Attribution License (http://creativecommons.org/licenses/by/2.0), which permits unrestricted use, distribution, and reproduction in any medium, provided the original work is properly cited.
37.73 million metric tons in 2007 to 43.65 million metric tons in 2012 (http://www.fas.usda.gov/psdonline/psdreport. aspx?hidReportRetrievalName=BVS\&hidReportRetrievalID $=$ 708\&hidReportRetrievalTemplateID $=8$ ), the production of soybean oil is still insufficient to meet the consumer demand, and the low oil content in soybean seeds is the main factor restricting yield. Therefore, an increase in soybean oil yield is desirable and has been one of the most major goals of oilseed engineering.

To increase the production of soybean oil, it is not feasible to extend the planting area of the crop, because of increased competition for land by the rapidly rising population. Therefore, it is a better strategy to increase the seed oil content than to increase the planting area 
[1]. In recent years, molecular genetics approaches have been used to modify seed oil content. Over-expression of a diacylglycerol acyltransferase (AtDGAT) cDNA in wild-type $A$. thaliana enhanced oil deposition and average seed weight [2]. The research of Wang et al. indicated that the oil content of soybean seeds could be increased by upregulation of two soybean Dof-type transcription factor (GmDof) genes, which are associated with fatty acid biosynthesis [3]. The soybean genome also has been examined with regard to the relationships between gene expression profiles and gene function. Although the biochemical pathways that produce different storage components are well characterized, there is still no integrated model describing differentially expressed genes (DEGs) involved in soybean lipid biosynthesis. With the development of high-throughput technologies, including the newly developed Solexa/Illumina RNA-sequencing and DEG high-throughput deep sequencing approaches, new genes have been discovered and specific transcripts analyzed. In Severin's research, RNA Seq-Atlas provided a record of high-resolution gene expression in a set of various tissues. They also found dramatic tissue-specific gene expression of both the most highly-expressed genes and the genes specific to legumes in seed development and nodule tissues [4]. Furthermore, these technologies are useful for estimating overall gene expressions at different development stages and/or in different tissues, such as on rice transcriptome and disease [5-7].

In this study, we used Illumina Solexa sequencing to investigate gene expression in soybean seeds at different developmental stages $(15,35,55$, and 65 days after flowering; DAF), and then compared transcript reads with the most recent release of the G. $\max$ genome sequence (assembly Glyma1.01). Illumina Solexa de novo sequencing technology identified a total of 11592,16594 , and 16255 differentially expressed unigenes between the 15 and 35 DAF seeds, the 15 and 55 DAF seeds, and the 15 and 65 DAF seeds, respectively (Additional file 1: Tables S1, Additional file 2: Table S2, Additional file 3: Table: S3). Of these, 9905 unigenes existed in all three of these contrast groups and represented co-expressed unigenes. Furthermore, 124 candidate unigenes were screened from the three contrasting cDNA libraries and characterized as those responsible for lipid synthesis. Our researches provide the whole picture of DEG transcription patterns and expression levels during soybean seed development. The elucidation of DEG transcription patterns at specific stages of seed development also lays the foundation for understanding the molecular mechanisms underlying oil production. This research provides direction for controlling genes over-expression to increase soybean oil content.

\section{Methods}

\section{Plant culture and collection}

The soybean cultivar Jiyu-72 was used as the experimental material. The plants were grown in a green house with a 15 -h light $\left(200 \mu \mathrm{Em}^{-2} \mathrm{~s}^{-1}, 25^{\circ} \mathrm{C}\right) / 9$-h dark photoperiod $\left(23^{\circ} \mathrm{C}\right)$, with relative humidity controlled at $75 \%$. The developmental processes of soybean seeds from flowering to seed maturity were observed from July to October 2010. Pods were harvested at 15 DAF (immature stage), and then every 5 days until 70 DAF (pods containing mature seeds). After removing the seed coat, the seeds were used for oil extraction or frozen at $-80^{\circ} \mathrm{C}$ until mRNA extraction and sequencing.

\section{Measurement of oil content}

To extract the oil (or lipid), seeds harvested at 15, 20, $25,30,35,40,45,50,55,60,65$, and 70 DAF were ovendried to constant weight at $85^{\circ} \mathrm{C}$. The dry samples were ground to a fine powder in a disintegrator, and the powder was transferred into $10-\mathrm{mL}$ glass tubes for oil extraction. Oil was extracted using ligarine to determine total lipids (TL) gravimetrically [8]. Oil extraction was performed using a SER148 3/6 Extraction apparatus (VELP Scientifica, Italy). Extractions were carried out using triplicate samples for each stage, and mean values were determined. Errors are shown as standard deviations.

\section{Total RNA extraction, library construction, and deep sequencing}

RNA was isolated from the seeds harvested at 15, 35, 55, and 65 DAF, respectively, using a TIANGEN RNA Prep Pure Plant kit (Tiangen Biotech Co. Ltd., Beijing, China) following the manufacturer's protocol, followed by a chloroform extraction to improve RNA purity. The yield and quality of total RNA samples were determined using a ND-1000 NanoDrop spectrophotometer. For RNA library construction and deep sequencing, equal quantities of RNA samples from seeds at the four developmental stages were pooled. Approximately $6 \mu \mathrm{g}$ RNA representing each group was submitted to Solexa (now Illumina Inc.) for sequencing.

\section{Differential expression (DE) detection}

The raw data were filtered to remove adaptor reads, low quality reads, and reads of copy number $=1$, yielding a dataset consisting of clean reads. For the tissue-specific analyses, raw digital gene expression data were normalized using a variation of the reads/Kb/million (RPKM) method $[9,10]$. The RPKM method corrects for biases in total gene exon size and normalizes for the total short read sequences obtained in each tissue library. Genes with data P-value $<0.005$, false discovery rate (FDR) $\leq 0.001$, and estimated absolute $\log _{2}$-fold change $\geq 1$ in 
sequence counts across libraries were considered to be significantly differentially expressed. Differentially expressed genes that were co-expressed during these four stages were then subjected to cluster analysis using the $\mathrm{R}$ program (a language and environment for statistical computing and graphics). Functional categorization of stress-regulated genes was performed using BGI WEGO (http://wego.genomics.org.cn/cgi-bin/wego/index.pl).

\section{Quantitative real-time PCR (qRT-PCR) analysis}

To verify the data obtained by Solexa RNA-seq, qRTPCR was performed on 12 genes with $\log _{2}$ ratios ranging from 2 to 11 (Additional file 4: Table S4). The RNA samples used for the qRT-PCR assays were the same as those used for the DEG experiments. qRT-PCR was performed on a Mx3000P instrument (Stratagene), with SYBR-Green detection (Quant qRT-PCR Kit, Tiangen Biotechnology), according to the manufacturer's instructions. The soybean tubulin gene was used as an internal control (forward primer: 5'ATGTTCCGTGGCAAGA TGAG-3', reverse primer: 5'-CATTGTTGGGAATCCAC TC-3') [11]. Primers were designed using the Primer Premier 5.0 and Oligo 6 programs to amplify products approximately 150-bp long. Thermal cycle conditions were as follows: $2 \mathrm{~min}$ at $95^{\circ} \mathrm{C}$ followed by 40 cycles of $15 \mathrm{~s}$ at $95^{\circ} \mathrm{C}, 15 \mathrm{~s}$ at $56-57^{\circ} \mathrm{C}$, and $15 \mathrm{~s}$ at $72^{\circ} \mathrm{C}$. Each cDNA was analyzed three times, after which the average threshold cycle $(\mathrm{Ct})$ was calculated per sample. Standard curves were established for all investigated genes using a series of amplicon dilutions. The relative expression levels were calculated as $2^{-}$( $\Delta \mathrm{Ct}$ of $35,55,65 \mathrm{DAF}-\Delta \mathrm{Ct}$ of $\left.15 \mathrm{DAF}\right)$.

\section{Results}

\section{Accumulation of oil at different stages of seed development}

To explore lipid accumulation during development of soybean seeds, we quantified lipid content in soybean seeds harvested from 15 to 70 DAF. As shown in Figure 1, the oil content began to increase from 15 DAF, then markedly increased from 15 to $20 \mathrm{DAF}$, and then remained stable from 20 to 25 DAF before again increasing rapidly from 25 to 35 DAF. From 35 to 55 DAF, the oil content showed a steady increase, peaking at 60 DAF before dropping at 65 DAF. These results indicated that for the soybean cultivar JiYu-72, 15 DAF marked the beginning of oil accumulation, 35 DAF was the key stage for the rapid increase in the oil content. 55 DAF was the stage at which oil content was high and stable, and 65 DAF was the stage at which oil content decreased and nearly unchanged. Therefore, in order to explore the differentially expressed genes associated with lipid biosynthesis during soybean seed development, the following sequencing and qRT-PCR

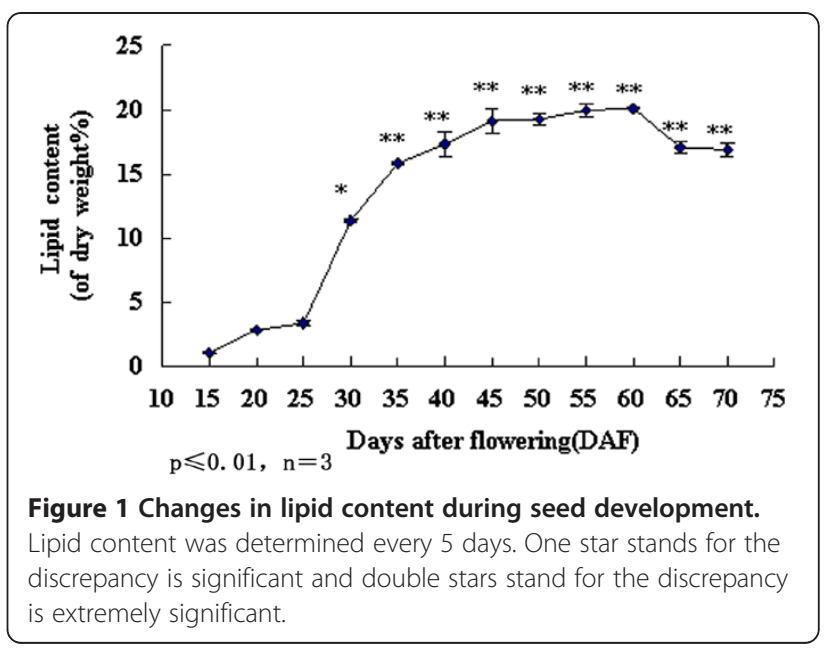

analyses were performed using samples from these four different developmental stages.

\section{Screening of differentially expressed genes (DEGs) from massive datasets}

Initially, Solexa RNA-sequencing revealed a total of 11592, 16594, 16255 differentially expressed unigenes between 35 DAF and 15 DAF seeds, 55 DAF and 15 DAF seeds, and 65 DAF and 15 DAF seeds, respectively (Table 1). Among them, 9905 unigenes were present in all three of these contrast groups, and 1687, 6689, and 6350 were differentially expressed only in the 35 vs 15 DAF, 55 vs 15 DAF, and 65 vs 15 DAF groups, respectively. From the large datasets, we found that down-regulated genes were more abundant than up-regulated genes during soybean seed development. The normalized data are shown as scatter plots of $\log _{10}$-transformed transcription counts for the four different samples (Additional file 5: Figure S1).

There were 680, 1004, and 850 up-regulated unigenes and 10912, 15590, and 15405 down-regulated unigenes in the three respective groups (Table 1). Among the upregulated unigenes, 352 were co-expressed genes showing increases of at least 2 -fold $\left(\log _{2}\right.$ ratio $\left.\geq 1\right)$, and 269 , 258 , and 65 of the up-regulated genes were specific to each of the three respective groups. Among the downregulated unigenes, 9476 were co-expressed genes showing decreases of at least 2 -fold $\left(\log _{2}\right.$ ratio $\left.\leq 1\right)$, and 672 , 1278 , and 769 of the down-regulated genes were specific to each of the three respective groups (Figure 2).

Differentially expressed genes provide clues about the molecular events related to seed development. Further investigation of highly expressed genes may be warranted to determine what functional roles the highly expressed sequences may play in soybean seeds. To understand the relationship between the time at which unigenes are co-expressed and their biological 
Table 1 Differentially expressed genes between seeds of 35 DAF and 15 DAF, 55 DAF and 15 DAF, and 65 DAF and 15 DAF

\begin{tabular}{|c|c|c|c|c|c|c|c|}
\hline \multirow[t]{3}{*}{ Contrast groups } & \multicolumn{7}{|c|}{ No. of genes } \\
\hline & \multirow{2}{*}{$\begin{array}{c}\text { Total } \\
\text { genes }\end{array}$} & \multirow{2}{*}{$\begin{array}{l}\text { Co-expressed } \\
\text { genes }\end{array}$} & \multirow{2}{*}{$\begin{array}{c}\text { Distinct } \\
\text { genes }\end{array}$} & \multicolumn{2}{|c|}{ Up-regulated } & \multicolumn{2}{|c|}{ Down-regulated } \\
\hline & & & & Known $^{a}$ & Unknown $^{\mathbf{b}}$ & Known $^{a}$ & Unknown $^{\mathrm{b}}$ \\
\hline Seeds of 35DAF-VS-15DAF & 11592 & 9905 & 1687 & 419 & 261 & 7314 & 3598 \\
\hline Seeds of 55DAF-VS-15DAF & 16594 & 9905 & 6689 & 646 & 358 & 10203 & 5378 \\
\hline Seeds of 65DAF-VS-15DAF & 16255 & 9905 & 6350 & 529 & 321 & 10246 & 5159 \\
\hline
\end{tabular}

"known" represents genes with Gene Ontology annotation.

'unknown' ${ }^{\text {b' }}$ represents genes without Gene Ontology annotation.

significance, we carried out Gene Ontology enrichment analysis.

\section{Gene ontology category analysis and DEGs in each category}

As a useful tool for gene functional annotation, WEGO (Web Gene Ontology Annotation Plot) has been widely used in many important studies, including the rice genome project and the silkworm genome project [12-14]. It has become one of the most commonly used tools for downstream gene annotation analysis, especially in comparative genomics studies. In this research, WEGO analysis assigned the DEGs (FDR $\leq 0.001$ and $\mid \log _{2}$ ratio $\mid \geq 1$ ) to three functional categories; Cellular Component, Molecular Function, or Biological Process. A total of 11963 unigenes had at least one GO functional category. In the three contrast groups, all differentially expressed genes with a Gene Ontology annotation were further classified into subsets. There were 11 subsets within the Cellular Component category, 10 subsets within the Molecular Function category, and 23 subsets within the Biological Process category (Figure 3). Thus, the most abundant unigenes were related to binding and catalytic activity in the Molecular Function category, cell, cell parts and organelles in the Cellular Component category, and cellular and metabolic in the Biological Process category.
The results showed that these types of genes were highly enriched in our soybean transcriptomes. In terms of the categories, the three contrast groups showed similar patterns.

Because we are interested in lipid biosynthesis, we focused on genes associated with this process that were differentially expressed during seed development. From the Component Ontology data, eight differentially coexpressed lipid particle unigenes were identified. Among them, seven unigenes were up-regulated, and one unigene was down-regulated during seed development. From the Function Ontology data, unigenes associated with lipid biosynthesis could be further classified into three categories: those with lipid kinase activity (9 differentially co-expressed down-regulated unigenes); those with fatty acid ligase activity (6 differentially co-expressed down-regulated unigenes); and those with fatty acid synthase activity (18 differentially co-expressed unigenes, 4 of which were up-regulated and 14 down-regulated). From the Process Ontology data, unigenes associated with lipid biosynthesis were in two categories; lipid biosynthetic process (78 differentially co-expressed unigenes, 5 of which were up-regulated, and 73 down-regulated), and fatty acid biosynthetic process (49 differentially coexpressed unigenes, 4 of which were up-regulated, and 45 down-regulated). Interesting, the 49 co-expressed
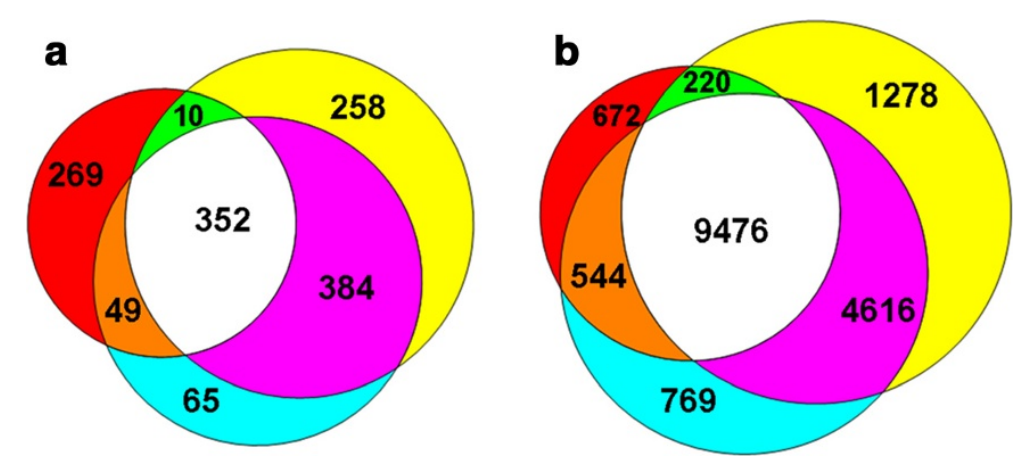

Figure 2 Venn diagram showing overlaps among up-regulated genes during soybean seed development. (a). All the unigenes that showed increases of at least 2-fold $\left(\log _{2}\right.$ ratio $\left.\geq 1\right)$ in the 3 contrasts, $(\mathbf{b})$. All the unigenes that showed decreases of at least 2 -fold $\left(\log _{2}\right.$ ratio $\left.\leq 1\right)$ in the 3 contrasts. Number in one circle denotes stage-specific genes, and number in two or more intersecting circles denotes overlapped genes. 

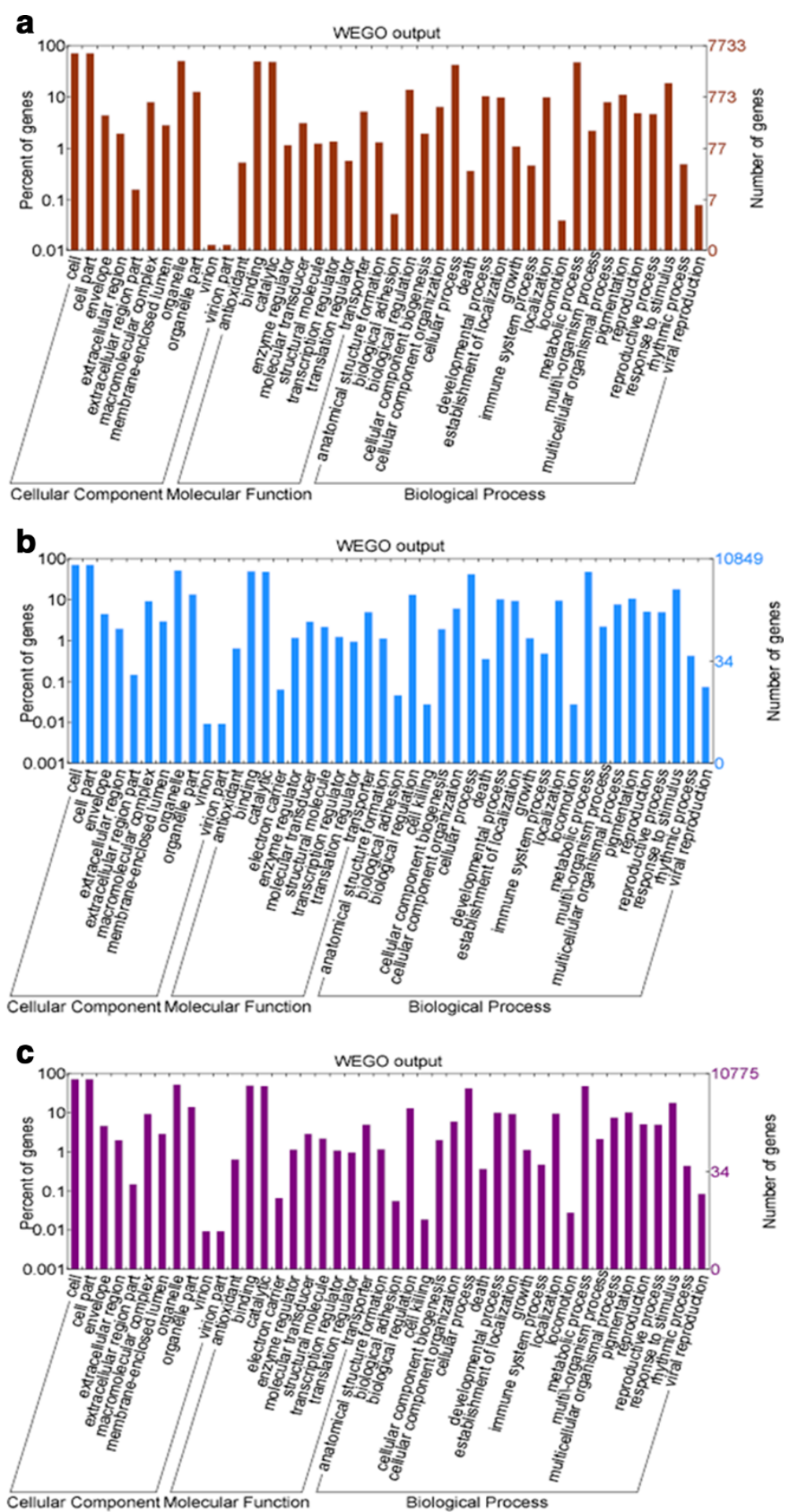

Figure 3 Functional categorization of significantly differentially expressed genes during seed development. Functional categorization was performed using BGI WEGO (Web Gene Ontology Annotation Plot). (a). The functional categorization of differentially expressed genes between $35 \mathrm{DAF}$ and $15 \mathrm{DAF}$. (b). The functional categorization of differentially expressed genes between 55 DAF and 15 DAF. (c). The functional categorization of differentially expressed genes between 65 DAF and 15 DAF. 
Table 2 DEGs identity, expression ratios, and Gene Ontology annotations

\begin{tabular}{|c|c|c|c|c|c|c|}
\hline \multirow[t]{2}{*}{ Unigene ID } & \multirow[t]{2}{*}{ Gene ID } & \multirow{2}{*}{$\begin{array}{l}\text { Accession } \\
\text { No }\end{array}$} & \multirow[t]{2}{*}{ Annotation } & \multicolumn{3}{|c|}{$\log _{2}$ ratio } \\
\hline & & & & 35 DAF & $55 \mathrm{DAF}$ & 65 DAF \\
\hline gnl|UG|Gma\#S15927547 & 31463931 & CD405959 & Catalytic activity, mRNA sequence & -12.45 & -12.45 & -12.45 \\
\hline gnl|UG|Gma\#S15936780 & 31473219 & CD415247 & $\begin{array}{l}\text { Gm_ck5311 Soybean induced by } \\
\text { salicylic acid G. max CDNA 3' }\end{array}$ & -1.52 & -5.95 & -13.86 \\
\hline gnl|UG|Gma\#S21537460 & 51335477 & Co979343 & Nucleoside-triphosphatase activity & -1.69 & -2.00 & -1.27 \\
\hline gnl|UG|Gma\#S21568089 & 51344862 & CO985595 & Intracellular membrane-bounded organelle & -1.85 & -14.73 & -4.59 \\
\hline gnl|UG|Gma\#S21568290 & 51344703 & Co985436 & GM89024B1 A03.r1 Gm-r1089 G. max CDNA & -2.16 & -2.20 & -3.15 \\
\hline gnl|UG|Gma\#S23063462 & 58016474 & CX703216 & $\begin{array}{l}\text { Water stressed } 48 \mathrm{~h} \text { segment } 2 \text { gmrtDrNS01 } \\
\text { G. max CDNA 3' }\end{array}$ & -1.81 & -1.39 & -1.76 \\
\hline gnl|UG|Gma\#S23071366 & 58024339 & CX711080 & Oxidoreductase activity & -8.01 & -11.12 & -11.05 \\
\hline gnl|UG|Gma\#S39303981 & 151397976 & EV267849 & Carbon-carbon lyase activity & -6.22 & -5.33 & -13.86 \\
\hline gnl|UG|Gma\#S39304525 & 151398522 & EV268393 & GLMA586TF JCVI-SOY2 G. max CDNA 5' & -4.60 & -2.39 & -2.32 \\
\hline gnl|UG|Gma\#S39310377 & 151405779 & EV274245 & GLMC444TF JCVI-SOY2 G. max CDNA 5' & -2.62 & -1.68 & -2.04 \\
\hline gnl|UG|Gma\#S39548296 & 152926331 & EU003576 & Oxidoreductase activity & -6.08 & -10.80 & -10.22 \\
\hline gnl|UG|Gma\#S45535109 & 192301009 & FG990109 & GLLD114TF JCVI-SOY1 G. max CDNA 5' & -1.60 & -1.97 & -2.13 \\
\hline gnl|UG|Gma\#S45548971 & 192312746 & FK003972 & GLMDD92TR JCVI-SOY2 G. max cDNA 5' & -1.23 & -1.07 & -1.57 \\
\hline gnl|UG|Gma\#S45553331 & 192318756 & FK008332 & Oxidoreductase activity & -4.38 & -2.62 & -6.01 \\
\hline gnl|UG|Gma\#S46507969 & 193399415 & FK361305 & $\begin{array}{l}\text { Soybean Seeds Containing Globular-Stage } \\
\text { Embryos G. max CDNA, mRNA sequence }\end{array}$ & -2.95 & -4.39 & -14.46 \\
\hline gnl|UG|Gma\#S46512605 & 193402683 & FK365941 & Oxidoreductase activity & -3.98 & -4.68 & -5.61 \\
\hline gnl|UG|Gma\#S47453769 & 208298984 & GE091511 & Oxidoreductase activity, mRNA sequence & -3.28 & -14.04 & -14.04 \\
\hline gnl|UG|Gma\#S47698257 & 209701642 & BW673128 & Oxidoreductase activity, mRNA sequence & -3.89 & -13.83 & -4.34 \\
\hline gnl|UG|Gma\#S48312113 & 210145949 & AK244640 & Oxidoreductase activity & -2.54 & -4.24 & -2.95 \\
\hline gnl|UG|Gma\#S48312226 & 210145836 & AK244527 & Oxidoreductase activity & -1.22 & -2.76 & -2.62 \\
\hline gnl|UG|Gma\#S48312292 & 210145770 & AK244461 & G. max CDNA & -5.08 & -14.02 & -5.71 \\
\hline gnl|UG|Gma\#S48312360 & 210145702 & AK244393 & G. max cDNA & -7.45 & -10.67 & -9.92 \\
\hline gnl|UG|Gma\#S48312476 & 210145586 & AK244277 & G. max cDNA & -2.29 & -2.99 & -2.62 \\
\hline gnl|UG|Gma\#S48312873 & 210145189 & AK243880 & G. max cDNA & -5.00 & -7.34 & -7.85 \\
\hline gnl|UG|Gma\#S48313629 & 210144071 & AK286853 & Oxidoreductase activity & -5.01 & -6.81 & -6.93 \\
\hline gnl|UG|Gma\#S48313665 & 210144035 & AK286817 & Oxidoreductase activity & -1.60 & -2.61 & -2.20 \\
\hline gnl|UG|Gma\#S48314836 & 210142864 & AK285646 & Oxidoreductase activity & -5.68 & -10.31 & -10.25 \\
\hline gnl|UG|Gma\#S48315365 & 210142183 & AK246102 & G. max cDNA & -12.13 & -2.15 & -2.09 \\
\hline gnl|UG|Gma\#S48315795 & 210141753 & AK245672 & Threonine kinase activity & -1.11 & -1.38 & -1.25 \\
\hline gnl|UG|Gma\#S48315845 & 210141703 & AK245622 & Fatty acid ligase activity & -4.52 & -5.95 & -5.30 \\
\hline gnl|UG|Gma\#S48316028 & 210141520 & AK245439 & Fatty acid synthase activity & -2.84 & -6.12 & -6.05 \\
\hline gnl|UG|Gma\#S48316351 & 210141197 & AK245114 & Fatty acid synthase activity & -2.05 & -11.17 & -2.90 \\
\hline gnl|UG|Gma\#S48316398 & 210141150 & AK245067 & G. max CDNA & -2.16 & -3.24 & -3.39 \\
\hline gnl|UG|Gma\#S48316494 & 210141054 & AK244973 & Oxidoreductase activity & -4.06 & -3.80 & -3.91 \\
\hline gnl|UG|Gma\#S48316776 & 210140772 & AK244691 & G. max cDNA & -1.54 & -2.50 & -2.62 \\
\hline gnl|UG|Gma\#S48540273 & 213602571 & DB978070 & Transferase activity, mRNA sequence & -11.98 & -11.98 & -11.98 \\
\hline gnl|UG|Gma\#S4876535 & 6070118 & AW099690 & Oxidoreductase activity & -12.38 & -12.38 & -12.38 \\
\hline gnl|UG|Gma\#S4928292 & 7924555 & AW830581 & G. max cDNA clone & -1.09 & -1.79 & -1.06 \\
\hline gnl|UG|Gma\#S4989750 & 9983486 & BE657594 & oxidoreductase activity, mRNA sequence & -2.95 & -12.36 & -12.36 \\
\hline gnI|UG|Gma\#S4991939 & 10252627 & BE820393 & fatty acid ligase activity & -1.44 & -3.87 & -3.22 \\
\hline gnl|UG|Gma\#S5146402 & 505137 & D13949 & G. max lipoxygenase-2 mRNA & 8.72 & 7.41 & 8.23 \\
\hline
\end{tabular}


Table 2 DEGs identity, expression ratios, and Gene Ontology annotations (Continued)

\begin{tabular}{|c|c|c|c|c|c|c|}
\hline gnl|UG|Gma\#S5146516 & 2739009 & AF022464 & G. max cytochrome P450 monooxygenase & -2.15 & -3.03 & -3.11 \\
\hline gnl|UG|Gma\#S5146617 & 2270993 & AF004809 & G. max $\mathrm{Ca}+2$-bingding $\mathrm{EF}$ hand protein & 2.75 & 10.33 & 9.51 \\
\hline gnl|UG|Gma\#S5146661 & 18674 & X67304 & G. max mRNA for lipoxygenase-1 & 10.25 & 9.44 & 9.91 \\
\hline gnl|UG|Gma\#S5146662 & 1794171 & U50081 & G. max lipoxygenase-3 mRNA & 9.25 & 8.86 & 9.23 \\
\hline gnl|UG|Gma\#S5146890 & 43985 & U04785 & G. max Williams 82 lipoxygenase mRNA & -5.46 & -8.74 & -8.67 \\
\hline gnl|UG|Gma\#S53086049 & 255645378 & ВТ097946 & Unknown mRNA & -2.10 & -4.42 & -4.06 \\
\hline gnl|UG|Gma\#S53088884 & 255638127 & ВТ095104 & Unknown mRNA & -1.83 & -2.30 & -3.82 \\
\hline gnl|UG|Gma\#S53090173 & 255635620 & ВТ093810 & fatty acid synthase activity, unknown mRNA & -1.52 & -3.36 & -2.78 \\
\hline gnl|UG|Gma\#S52654816 & 254332339 & GR846384 & Monooxygenase activity & -2.25 & -2.78 & -3.29 \\
\hline gnl|UG|Gma\#S39313094 & 151407147 & EV276962 & $\begin{array}{l}\text { GLMCZ08TF JCVI-SOY2,G. max CDNA } \\
5 \text { ' mRNA sequence }\end{array}$ & -1.16 & -2.35 & -2.54 \\
\hline gnl|UG|Gma\#S53090215 & 255635538 & ВТ093768 & Oxidoreductase activity,unknown mRNA & -1.39 & -1.64 & -2.06 \\
\hline gnl|UG|Gma\#S4993394 & 10254082 & BE821848 & Fatty acid synthase activity, mRNA sequence & -1.21 & -2.19 & -1.78 \\
\hline gnl|UG|Gma\#S48316085 & 210141463 & AK245382 & UDP-glycosyltransferase activity & -1.63 & -2.06 & -1.84 \\
\hline gnl|UG|Gma\#S6669271 & 26046073 & CA783412 & Oxidoreductase activity, mRNA sequence & -3.45 & -6.81 & -4.05 \\
\hline gnl|UG|Gma\#S48315123 & 210142573 & AK285451 & Catalytic activity & -1.51 & -6.89 & -4.90 \\
\hline gnl|UG|Gma\#S48313908 & 210143792 & AK286574 & G. max CDNA & -2.07 & -3.73 & -3.34 \\
\hline gnl|UG|Gma\#S48316204 & 210141408 & AK245263 & Oxidoreductase activity & -1.32 & -2.34 & -2.35 \\
\hline gnl|UG|Gma\#S39303626 & 151397621 & EV267494 & Oxidoreductase activity & -1.05 & -1.32 & -1.06 \\
\hline gnl|UG|Gma\#S48316577 & 210140971 & AK244890 & Methyltransferase activity & -1.22 & -3.23 & -2.49 \\
\hline gnl|UG|Gma\#S32218187 & 90658387 & DQ394572 & $\begin{array}{l}\text { G. max cytochrome P450 monooxygenase } \\
\text { CYP97C10 (CYP97C10) mRNA }\end{array}$ & -3.05 & -1.25 & -2.12 \\
\hline gnl|UG|Gma\#S48314962 & 210142734 & AK285227 & Cyclase activity & -2.25 & -3.28 & -2.84 \\
\hline gnl|UG|Gma\#S53089425 & 255637080 & ВТ094559 & Unknown mRNA & -2.16 & -4.59 & -5.52 \\
\hline gnl|UG|Gma\#S52634774 & 254317397 & GR826342 & Oxidoreductase activity & -1.60 & -2.39 & -3.02 \\
\hline gnl|UG|Gma\#S21537312 & 51335213 & CO979079 & mRNA sequence, mRNA sequence & -3.48 & -5.92 & -4.96 \\
\hline gnl|UG|Gma\#S48316562 & 210140986 & AK244905 & G. max CDNA & -2.12 & -1.57 & -1.29 \\
\hline gnl|UG|Gma\#S23065186 & 58018200 & CX704942 & Monooxygenase activity & -5.00 & -12.44 & -12.44 \\
\hline gnl|UG|Gma\#S5146686 & 1399379 & U43683 & C-methyltransferase activity & -1.65 & -2.28 & -2.35 \\
\hline gnl|UG|Gma\#S53085203 & 255647037 & ВТ098796 & $\begin{array}{l}\text { C-4 methylsterol oxidase activity, } \\
\text { unknown mRNA }\end{array}$ & -1.24 & -2.26 & -1.68 \\
\hline gnl|UG|Gma\#S53086564 & 255644365 & ВТ097429 & Unknown mRNA & -1.45 & -2.73 & -3.21 \\
\hline gnl|UG|Gma\#S48313496 & 210144204 & AK286986 & G. max CDNA & -1.70 & -3.40 & -3.21 \\
\hline gnl|UG|Gma\#S53089772 & 255636400 & ВТ094211 & $\begin{array}{l}\text { Geranyltranstransferase activity, } \\
\text { unknown mRNA }\end{array}$ & -1.62 & -1.73 & -2.25 \\
\hline gnl|UG|Gma\#S39313896 & 151407949 & EV277764 & UDP-galactosyltransferase activity & -3.83 & -5.75 & -14.29 \\
\hline gnl|UG|Gma\#S52663531 & 254341371 & GR855099 & Small conjugating protein ligase activity & -1.65 & -1.50 & -2.21 \\
\hline gnl|UG|Gma\#S53088132 & 255639591 & ВТ095858 & Unknown mRNA & 1.19 & -1.17 & -1.02 \\
\hline gnl|UG|Gma\#S53085581 & 255646289 & ВТ098415 & $\begin{array}{l}\text { 3-hydroxyacyl-[acyl-carrier-protein] } \\
\text { dehydratase activity unknown mRNA }\end{array}$ & -2.63 & -5.89 & -5.38 \\
\hline gnl|UG|Gma\#S30676923 & 85001710 & DQ340246 & $\begin{array}{l}\text { G. max cytochrome P450 monooxygenase } \\
\text { CYP90A15 (CYP90A15) mRNA }\end{array}$ & -2.18 & -4.17 & -3.39 \\
\hline gnl|UG|Gma\#S5142779 & 22931631 & BU548770 & Gm-r1088 G. max cDNA clone Gm-r1088-6501 3' & -4.74 & -7.44 & -7.37 \\
\hline gnl|UG|Gma\#S39312371 & 151406424 & EV276239 & Phosphatidylinositol phosphate kinase activity & -4.60 & -13.22 & -13.22 \\
\hline gnl|UG|Gma\#S22952836 & 57576101 & CX549072 & Lipid kinase activity & -1.46 & -2.12 & -2.30 \\
\hline gnl|UG|Gma\#S46837027 & 193692332 & FK631119 & Lipid kinase activity & -2.54 & -2.17 & -4.90 \\
\hline gnl|UG|Gma\#S23071197 & 58024263 & CX711004 & Lipid kinase activity & -1.34 & -1.55 & -1.81 \\
\hline
\end{tabular}


Table 2 DEGs identity, expression ratios, and Gene Ontology annotations (Continued)

\begin{tabular}{|c|c|c|c|c|c|c|}
\hline gnl|UG|Gma\#S21568721 & 51345091 & CO985824 & Phosphatidylinositol phosphate kinase activity & -2.00 & -2.25 & -2.70 \\
\hline gnl|UG|Gma\#S47246628 & 208083682 & GD884374 & $\begin{array}{l}\text { Soybean Seeds Containing Globular-Stage } \\
\text { Embryos G. max cDNA mRNA sequence }\end{array}$ & -2.65 & -3.77 & -2.38 \\
\hline gnl|UG|Gma\#S23061755 & 58014763 & CX701505 & Phosphatidylinositol phosphate kinase activity & -2.37 & -4.07 & -2.51 \\
\hline gnl|UG|Gma\#S48551001 & 214004471 & DB984819 & Lipid kinase activity, mRNA sequence & -1.83 & -3.59 & -3.28 \\
\hline gnl|UG|Gma\#S18957041 & 42723029 & CK768928 & Phosphoinositide 3-kinase activity & -1.27 & -1.40 & -1.05 \\
\hline gnl|UG|Gma\#S4989748 & 9983484 & BE657592 & CoA-ligase activity & -5.06 & -16.02 & -16.02 \\
\hline gnI|UG|Gma\#S39311670 & 151405724 & EV275538 & Fatty acid ligase activity, mRNA sequence & -3.25 & -2.95 & -2.88 \\
\hline gnl|UG|Gma\#S45544712 & 192308617 & FG999712 & Fatty acid ligase activity & -2.12 & -12.47 & -4.75 \\
\hline gnl|UG|Gma\#S4994916 & 10255604 & BE823370 & $\begin{array}{l}\text { Gm-r1070 G. max cDNA clone Gm-r1070-7609 } \\
\text { 3'mRNA sequence }\end{array}$ & -1.10 & -14.60 & -4.11 \\
\hline gnl|UG|Gma\#S53085813 & 255645838 & ВТ098182 & Unknown mRNA & 8.39 & 10.22 & 9.95 \\
\hline gnl|UG|Gma\#S22952816 & 57576081 & CX549052 & Fatty acid synthase activity & -1.25 & -1.67 & -2.11 \\
\hline gnl|UG|Gma\#S48312148 & 210145914 & AK244605 & Fatty acid synthase activity & -1.30 & -3.09 & -3.12 \\
\hline gnl|UG|Gma\#S53090057 & 255635847 & ВТ093926 & Unknown mRNA, fatty acid synthase activity & 6.68 & 7.34 & 7.40 \\
\hline gnl|UG|Gma\#S18957150 & 42723162 & CK769061 & Fatty acid synthase activity, mRNA sequence & 4.08 & 6.59 & 6.11 \\
\hline gnl|UG|Gma\#S48312652 & 210145410 & AK244101 & Acyl-[acyl-carrier-protein] hydrolase activity & -1.39 & -2.41 & -2.69 \\
\hline gnl|UG|Gma\#S5129103 & 16345261 & B1970856 & Fatty acid synthase activity & -1.51 & -4.84 & -4.01 \\
\hline gnl|UG|Gma\#S48315227 & 210142469 & AK285347 & Fatty acid synthase activity & -1.24 & -3.64 & -3.25 \\
\hline gnl|UG|Gma\#S53090055 & 255635851 & ВТ093928 & Fatty acid synthase activity unknown mRNA & -2.14 & -1.37 & -2.12 \\
\hline gnl|UG|Gma\#S5146394 & 7862173 & AF260565 & $\begin{array}{l}\text { G. max beta-ketoacyl-acyl carrier protein } \\
\text { synthase III mRNA }\end{array}$ & -1.10 & -2.05 & -2.09 \\
\hline gnl|UG|Gma\#S5146406 & 7385202 & AF243183 & G. max beta-ketoacyl-ACP synthetase I-2 mRNA & -1.64 & -3.36 & -3.78 \\
\hline gnl|UG|Gma\#S23063275 & 58016255 & CX702997 & Fatty acid synthase activity & -1.48 & -1.89 & -2.94 \\
\hline gnl|UG|Gma\#S48316073 & 210141475 & AK245394 & G. max CDNA & 6.81 & 7.52 & 7.48 \\
\hline gnl|UG|Gma\#S4934087 & 6846492 & AW348782 & Fatty acid synthase activity, mRNA sequence & -3.49 & -5.72 & -4.91 \\
\hline gnl|UG|Gma\#S45541550 & 192306090 & FG996550 & Lipid particle & 4.21 & 3.89 & 3.84 \\
\hline gnl|UG|Gma\#S48531444 & 213596804 & DB978036 & Lipid particle & 4.87 & 5.13 & 4.79 \\
\hline gnl|UG|Gma\#S48316140 & 210141408 & AK245327 & Lipid particle & -1.32 & -2.17 & -2.61 \\
\hline gnl|UG|Gma\#S18957317 & 42723121 & CK769020 & Lipid particle (GmPM13) mRNA & 3.75 & 3.86 & 4.05 \\
\hline gnl|UG|Gma\#S48312213 & 210145849 & AK244540 & Lipid particle & 5.29 & 5.12 & 5.03 \\
\hline gnl|UG|Gma\#S18956788 & 42722719 & CK768618 & Lipid particle & 4.63 & 4.51 & 4.48 \\
\hline gnl|UG|Gma\#S4995024 & 10255768 & BE823478 & G. max mRNA sequence & 8.05 & 8.80 & 8.79 \\
\hline gnl|UG|Gma\#S4899715 & 6747398 & AW317854 & Lipid particle & 4.64 & 4.75 & 4.74 \\
\hline
\end{tabular}

unigenes detected in the fatty acid biosynthetic process were all included in unigenes detected in the lipid biosynthetic process. Among the 113 unigenes analyzed by Gene Ontology, 15 co-expressed unigenes that showed high levels of expression ( $\log _{2}$ fold values $\geq 1$ ) could be responsible for lipid biosynthesis (Table 2).

Annotation of lipid-relevant genes in fatty acid pathway Genes usually interact with each other to carry out certain biological functions. Knowledge of the expressions of multiple genes and their regulation during oil biosynthesis is required to further understand the regulatory mechanisms controlling oil metabolism. Pathway- based analysis helps to clarify the biological functions of genes, and identifies significantly enriched metabolic pathways or signal transduction pathways associated with the DEGs compared with the whole genome background. For our research, 124 biological pathways, including the starch and sucrose metabolism pathway, the citrate cycle pathway, the fatty acid biosynthesis pathway, and many others were identified by KEGG pathway analysis of unigenes. A total of 4836, 6796, and 6758 DEGs with pathway annotations were identified in the three respective contrast groups. From those pathways, we selected the fatty acid biosynthesis pathway (Figure 4) for deep analysis. In the three contrast groups, 24 DEGs 


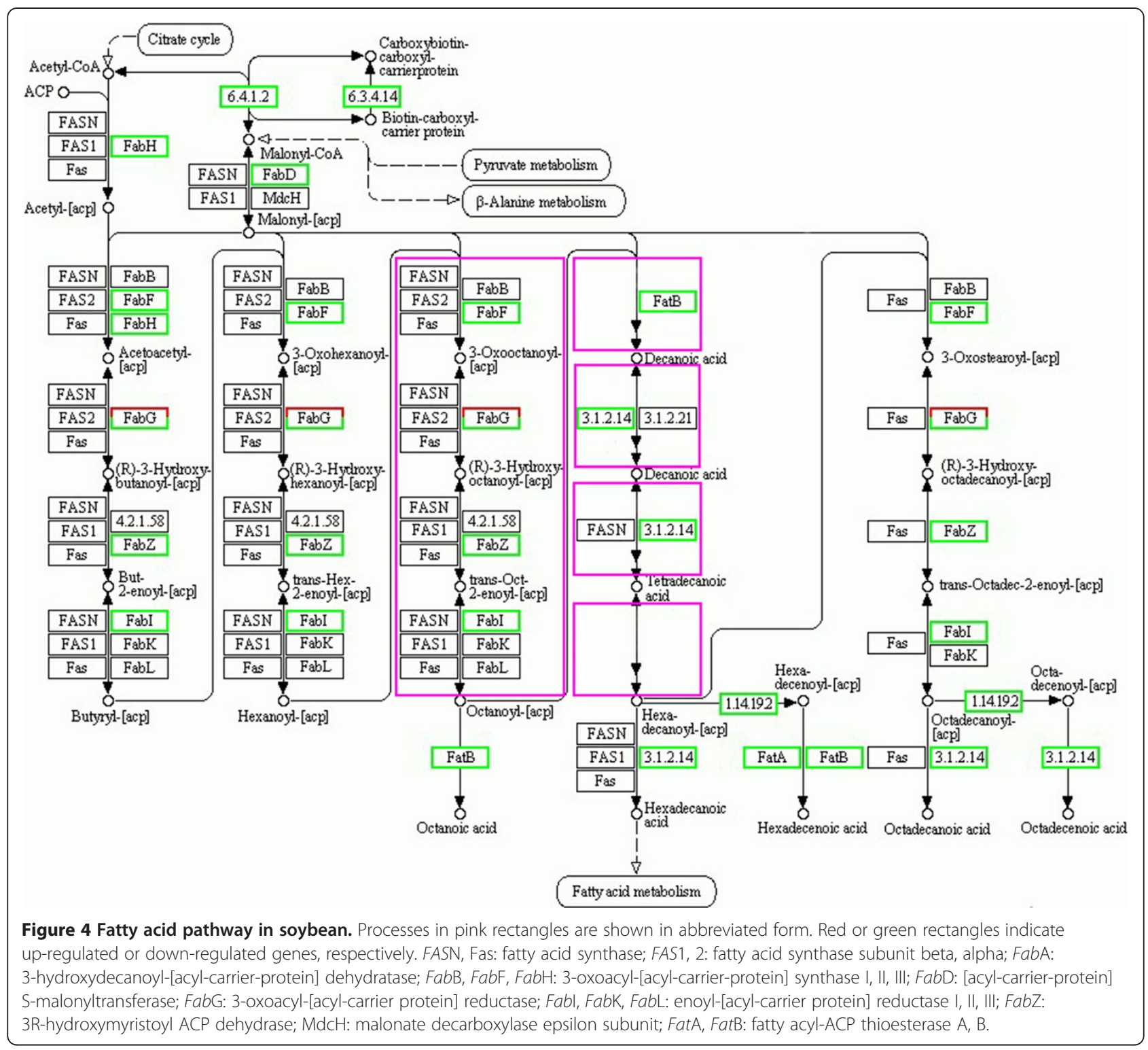

associated with the fatty acid pathway were coexpressed (Table 3). Interestingly, 13 of the identified unigenes were the same as those identified from the GO analysis. Among those 24. DGEs, only four ungenes (BT098182, AK245394, BT093926, BT093412) were upregulated, which are encoding 3-oxoacyl-[acyl-carrier protein] reductases $(F a b G)$ and were annotated as having fatty acid synthase activity. The other 20 unigenes were down-regulated, which may be the negatively controlled genes in the fatty acid biosynthesis pathway. Figure 4 shows the locations of the differentially coexpressed unigenes in the fatty acid pathway. Those shown in red and green represent genes that were upregulated and down-regulated, respectively, during seed development.

\section{Clustering of candidate genes}

A total of 124 unigenes, including those detected by GO and pathway analyses, were selected for clustering analysis. All of these unigenes showed different expression profiles. The genes were clustered according to the similarity of their expression patterns. A subset of the data is shown in Figure 5. The heatmap of the RPKM normalized $\log _{2}$-transformed transcription count was generated using $\mathrm{R}$ programs. Up-regulated unigenes are shown in red, while down regulated ones are shown in green. This analysis allowed us to define common qualitative patterns in gene expression changes over time. Our results suggested that only a few genes were expressed differentially in the three contrast groups, and that the majority of the transcriptome had approximately similar 
Table 3 DEGs involved in fatty acid pathway

\begin{tabular}{|c|c|c|c|c|c|c|}
\hline \multirow[t]{2}{*}{ Unigene ID } & \multirow[t]{2}{*}{ Gene ID } & \multirow[t]{2}{*}{ Accession No } & \multirow[t]{2}{*}{ Annotation } & \multicolumn{3}{|c|}{$\log _{2}$ ratio } \\
\hline & & & & $35 \mathrm{DAF}$ & $55 \mathrm{DAF}$ & $65 \mathrm{DAF}$ \\
\hline gnl|UG|Gma\#S53085813 & 255645838 & BT098182 & Unknown mRNA & 8.39 & 10.22 & 9.95 \\
\hline gnl|UG|Gma\#S48316073 & 210141475 & AK245394 & G. $\max$ cDNA & 6.81 & 7.52 & 7.48 \\
\hline gnl|UG|Gma\#S53090057 & 255635847 & ВТ093926 & Unknown mRNA, fatty acid synthase activity & 6.68 & 7.34 & 7.40 \\
\hline gnl|UG|Gma\#S53090570 & 255634845 & ВТ093412 & Catalytic activity, unknown mRNA & 3.88 & 5.09 & 4.29 \\
\hline gnI|UG|Gma\#S53086639 & 255642528 & ВТ097354 & Oxidoreductase activity, unknown mRNA & -10.89 & -10.89 & -10.89 \\
\hline nl|UG|Gma\#S53085581 & 255646289 & ВТ098415 & $\begin{array}{l}\text { 3-Hydroxyacyl-[acyl-carrier-protein] } \\
\text { dehydratase activity, unknown mRNA }\end{array}$ & -2.63 & -5.89 & -5.38 \\
\hline gnl|UG|Gma\#S4934087 & 6846492 & AW348782 & Fatty acid synthase activity, mRNA sequence & -3.49 & -5.72 & -4.91 \\
\hline gnl|UG|Gma\#S53089253 & 255637417 & ВТ094733 & Unknown mRNA & -2.01 & -4.98 & -4.68 \\
\hline gnl|UG|Gma\#S5129103 & 16345261 & BI970856 & Fatty acid synthase activity & -1.51 & -4.84 & -4.01 \\
\hline gnl|UG|Gma\#S48312866 & 210145196 & AK243887 & Metabolic process & -2.25 & -4.42 & -3.98 \\
\hline gnl|UG|Gma\#S5146406 & 7385202 & AF243183 & $\begin{array}{l}\text { G. max beta-ketoacyl-ACP } \\
\text { synthetase I-2 mRNA }\end{array}$ & -1.64 & -3.36 & -3.78 \\
\hline gnl|UG|Gma\#S5146689 & 1143321 & U40979 & $\begin{array}{l}\text { G. max alfa-carboxyltransferase (accA-2) } \\
\text { precursor mRNA }\end{array}$ & -1.19 & -5.05 & -3.66 \\
\hline gnl|UG|Gma\#S53087052 & 255641716 & ВТ096940 & Catalytic activity, unknown mRNA & -1.63 & -2.74 & -3.27 \\
\hline gnl|UG|Gma\#S48315227 & 210142469 & AK285347 & Fatty acid synthase activity & -1.24 & -3.64 & -3.25 \\
\hline gnl|UG|Gma\#S48312148 & 210145914 & AK244605 & Fatty acid synthase activity & -1.30 & -3.09 & -3.12 \\
\hline gnl|UG|Gma\#S23063275 & 58016255 & CX702997 & Fatty acid synthase activity & -1.48 & -1.89 & -2.94 \\
\hline gnl|UG|Gma\#S5146375 & 9621819 & AF165159 & $\begin{array}{l}\text { G. max carboxyl transferase alpha } \\
\text { subunit (accA-3) mRNA }\end{array}$ & -1.16 & -4.06 & -2.86 \\
\hline gnI|UG|Gma\#S48312652 & 210145410 & AK244101 & Acyl-[acyl-carrier-protein] hydrolase activity & -1.39 & -2.41 & -2.69 \\
\hline gnl|UG|Gma\#S53089095 & 255637722 & ВТ094891 & Unknown mRNA & -1.05 & -2.54 & -2.53 \\
\hline gnl|UG|Gma\#S48316546 & 210141002 & AK244921 & Oxidoreductase activity & -1.56 & -1.99 & -2.43 \\
\hline gnl|UG|Gma\#S48316068 & 210141480 & AK245399 & CoA carboxylase activity & -1.28 & -2.57 & -2.17 \\
\hline gnl|UG|Gma\#S22952816 & 57576081 & CX549052 & Fatty acid synthase activity & -1.25 & -1.67 & -2.11 \\
\hline gnl|UG|Gma\#S5146394 & 7862173 & AF260565 & $\begin{array}{l}\text { G. max beta-ketoacyl-acyl carrier } \\
\text { protein synthase III mRNA }\end{array}$ & -1.10 & -2.05 & -2.09 \\
\hline gnl|UG|Gma\#S23063462 & 58016474 & CX703216 & $\begin{array}{l}\text { Water-stressed } 48 \text { h segment } 2 \text { gmrtDrNS01 } \\
\text { G. max cDNA 3' }\end{array}$ & -1.81 & -1.39 & -1.76 \\
\hline
\end{tabular}

expression levels. In this manner, similar patterns were clustered together in the same manner as a taxonomic tree. Therefore, we can speculate on the roles of unigenes of unknown function by comparison to the annotated genes with known functions in the same cluster.

Confirmation of differentially expressed genes by qRT-PCR To confirm the expression patterns determined by Solexa RNA-sequencing analysis, we used qRT-PCR analyses to analyze expressions of 12 candidate genes (Additional file 4: Table S4). The soybean tubulin gene was used as an internal control. Although the Solexa $\log _{2}$-fold values showed slight variations compared with the corresponding values from the qRT-PCR analyses, the expression data from Solexa RNA-seq analysis was largely consistent with those obtained by qRT-PCR (Additional file 6: Figure S2).

\section{Discussion}

Lipid synthesis depends on the correct spatial and temporal activity of many gene products. These genes execute their function in three stages: fatty acid synthesis in the plastid, triacylglycerol (TAG) synthesis in the endoplasmic reticulum (ER), and assembly into an oil body. Significant improvements in oil accumulation must be accompanied by changes in activity of the genes involved in fatty acid biosynthesis in developing seeds. Identification of these genes and their regulatory pathway would provide not only new genetic information for understanding soybean seed development, but also for controlling gene expression in developing seeds to alter oil accumulation.

This study has provided a new data set identifying the expression of DEGs during oil accumulation in developing seeds. Massive parallel sequencing identified 11592, 


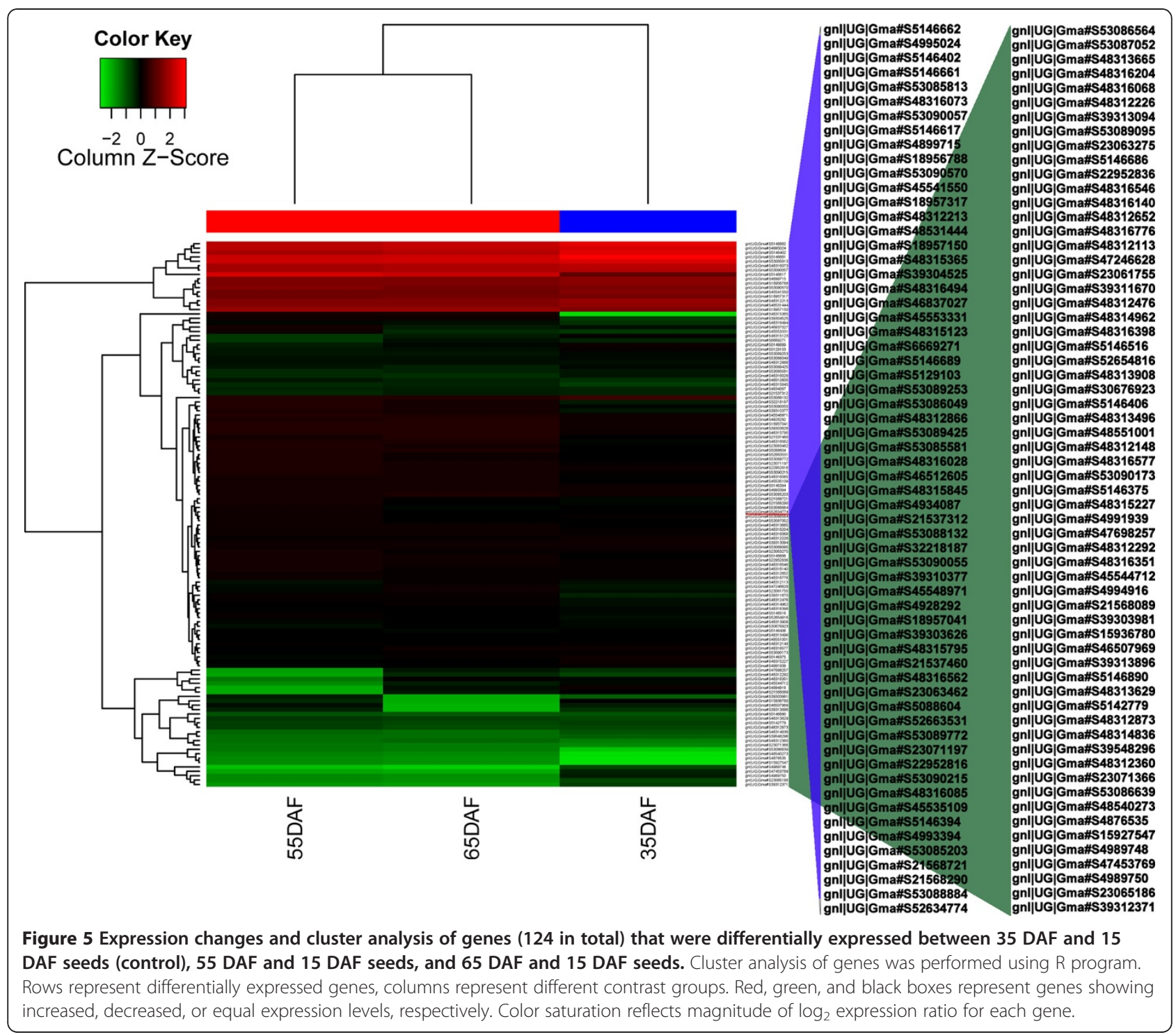

16594 and 16255 differentially expressed unigenes from three contrasting libraries covering four stages of seed development. We examined gene expression levels in detail, and found significant differences among the four growth stages. Among the differentially expressed genes, more were down-regulated than up-regulated, and some showed a differential expression pattern in all three contrast groups, indicating that there were overlaps at the transcriptional level. The fact that there were many down-regulated genes indicates that there are more negatively regulated genes than positively regulated ones with functions in the fatty acid pathway. However, it does not mean that lower expression of these genes leads to lower oil contents, because of the complexity of the lipid biosynthetic pathway. To identify the genes associated with lipid biosynthesis, we determined gene expression patterns at specific stages of seed development, and conducted a GO analysis. To explore the genes with unknown functions, the expression patterns of 124 unigenes were analyzed by hierarchical clustering according to similarities in expression profiles across all conditions.

In the lipid biosynthetic pathway, acetyl CoA carboxylase (ACCase) plays an important regulatory role. ACCase catalyzes the carboxylation of acetyl-CoA to malonyl-CoA [15], which is then transacylated by malonyl-CoA-acyl carrier protein transacylase $(F a b \mathrm{D}$ or $M C A T$,[EC 2.3.1.39]) to the acyl carrier protein (ACP), forming malonyl ACP. The latter adds a 2-carbon acetyl unit to the nascent or growing fatty acyl chain. The basic reaction of fatty acid synthesis is to combine molecules composed of two carbon units into longer chains to 
form fatty acids. The initial condensation of 2-carbon units is catalyzed by $\beta$-ketoacyl-ACP synthase III (KASIII, EC2.3.1.180) which uses acetyl-CoA and malonyl-ACP as substrates. After the two reductions and dehydration reactions, a 4-carbon fatty acid, butyrate, is produced. This is poorly condensed by KASIII but is a good substrate for $K A S I$, which elongates 4-carbon chains to 14carbon chains.

In this study, we studied in detail the genes with key roles in lipid biosynthesis. Very frequently, the same enzymatic function is redundantly encoded by several unigenes. This may be the result of different proteins referenced with the same EC number or it may represent different transcripts encoding specific enzyme subunits. This situation was significant in the present study. Most plants have two forms of ACCase, the homomeric form in the cytosol, composed of a single large polypeptide catalyzing the individual carboxylation reactions [16], and the heteromeric form in plastids, composed of four subunits; biotin carboxylase (BC) [17], biotin carboxyl carrier protein (BCCP) [18], $\alpha$-carboxyltransferase $(\alpha-C T)$ [19] and $\beta$ carboxyltransferase $(\beta-C T)$ [20]. In the present study, seven co-expressed unigenes (GenBank accession nos: BT094733, AK245399, U40979, AF165159, CX703216, BM188175, AW830581) encoding ACCases were identified as DEGs in the three contrast groups. All of them were downregulated $\left(\log _{2}\right.$ ratio $\left.\leq 1\right)$ in the three contrast groups except one gene (AK245356) encoding an ACCase was upregulated $\left(\log _{2}\right.$ ratio $\left.=1.4\right)$ at $35 \mathrm{DAF}$. Three co-expressed unigenes (GenBank accession nos: BM188175, CX703216, AW830581, EC6.3.4.14) that are associated with the transformation of BCCP to carboxybiotin-carboxyl-carrier protein were down-regulated during seed development.

The next enzyme in fatty acid pathway is FabD, which catalyzes the transfer of malonyl-CoA to the holo acyl carrier protein (ACP), generating malonyl-ACP [21]. In the present study, the co-expressed unigene (AW34878) encoding FabD was down-regulated during seed development.

$K A S I I I(F a b H)$ catalyzes the subsequent condensation and transacylation of acetyl-CoA with malonyl-ACP and has a universal role in fatty acid biosynthesis. Transgenic B. napus seeds overexpressing KASIII driven by napin also contained lower oil levels compared to what was found in the wild-type [22]. In the present study, the coexpressed unigene (AF260565) encoding KASIII was significantly down-regulated in the fatty acid pathway with a $\log _{2}$ ratio of $-1.10,-2.05$, and -2.09 in the three contrast groups. So compared to $15 \mathrm{DAF}$, the differentially expressed levels of the gene encoding KASIII at 35DAF, 55DAF, 65DAF negatively correlated to fatty acid synthesis during the seed development, which is consistent with previous research [23]. The same down-regulated patterns were also observed for FabF (AF243183,
CX702997, AK244605, AK285347), FabZ (BT098415), FabI (BI970856), FatB (AK244101) and oleoyl-[acyl-carrier-protein] hydrolase (EC 3.1.2.14, AK244101).

In most plant tissues, Acyl-ACP thioesterase is the major determinant of chain length and level of saturated fatty acids [24]. It plays an important role by influencing the fatty acid composition of the produced oil and then mainly the ratio of $16 \mathrm{C}$ to $18 \mathrm{C}$ fatty acids and the level of saturated fatty acid [25]. Two distinct but related thioesterase gene classes exist in higher plants: FatA is an acyl-specific thioesterase, with specificity for 18:1> $>18: 0>>16: 0$ fatty acids [26], which is considered an essential "housekeeping" enzyme in all plant cells; FatB is a thioesterase, which shows specificity for $16: 0>18: 1>18: 0$ fatty acids [27]. In this study, Solexa sequencing analysis showed that the expression of gene (CX706542) encoding FatA was unchanged at 35 DAF, but down-regulated at $55 \mathrm{DAF}$ and $65 \mathrm{DAF}$ with $\log _{2}$ ratios of -1.7 and -1.5 . While differentially co-expressed unigene (AK244101) encoding FatB showed constant decreased expressed levels with $\log _{2}$ ratios of $-1.4,-2.4$, -2.7 , respectively. Therefore, the expression level variations of CX706542 and AK244101 would influence the 16 and 18 carbon fatty acids synthesis, which needs to be confirmed by experiment.

Soybean seeds contain three lipoxygenase isozymes; lipoxygenases 1, 2, and 3. Lipoxygenases (linoleate: oxygen oxidoreductase; EC 1.13.11.12) catalyze the oxidation of unsaturated fatty acids to produce conjugated unsaturated fatty acid hydroperoxides, which are converted to volatile compounds associated with undesirable flavors [28]. Eliminating this enzyme from seeds could lead to better quality soybean protein and oil products. Three co-expressed lipoxygenase genes (X67304, U50081, D13949) identified in this study were among those with the highest expression levels. To our knowledge, this is the first report of a close correlation between lipoxygenase expression and fatty acid accumulation.

The P450 family is a large and diverse group of isozymes that mediate a diverse array of oxidative reactions. The activities of most of these enzymes are associated with biosynthetic processes such as phenylpropanoid, terpenoid, and fatty acid biosyntheses. Ten alkane-inducible $\mathrm{P} 450$ genes from Candida tropicalis (ATCC20336), which were responsible for omegahydroxylation of n-alkanes and fatty acids, were cloned [29]. In their research, these enzymes were believed to be at least in part limiting in the conversion of fatty acid to diacids, but their relative oxidative activity toward other fatty acids was not known. The two unigenes (AF022464, DQ340246) encoding soybean P450 monooxygenase were identified in this research. Both of them showed down-regulated expressions during 
seed development, which indicated that they are negatively correlated to the fatty acid accumulation. For the other differentially co-expressed unigenes shown in Table 2, there is little information available about their relationship with lipid accumulation. Their $\log _{2}$ ratios indicate that they have significant functions in regulating soybean seed development. However, our results cannot validate a direct relationship between these unigenes and oil accumulation. This topic requires further research.

Because we are interested in oil production in soybeans, we selected the fatty acid biosynthesis pathway for deep analysis from among the 124 biochemical pathways identified by Solexa sequencing. Twenty four coexpressed unigenes in the fatty acid pathway, including 13 that overlapped with the unigenes identified in the $\mathrm{GO}$ analysis, showed significant correlations with fatty acid accumulation (Table 3).

The up-regulated genes that were significantly correlated with fatty acid accumulation included ACCase and lipoxygenase. The down-regulated genes that were significantly correlated with fatty acid accumulation included FabD, KAS III, FabF, FabZ, FabI, FatB, FatA, P450, and oleoyl-[acyl-carrier-protein] hydrolase genes. FabG genes were both up- and down-regulated during seed development.

The analysis of the genes involved in the fatty acid biosynthetic pathway provides a basis to identify key regulatory processes controlling oil accumulation in soybean. However, biosynthetic pathways involve the cooperation of multiple genes. It is difficult to increase seed oil content by overexpressing a single gene. The large-scale characterization of unigenes described in this study shows comprehensive correlations between DEGs and fatty acid accumulation in soybean.

\section{Conclusion}

In this study, 11592, 16594, and 16255 differentially expressed unigenes were identified at 35,55 , and 65 days after flowering of soybean, respectively. Gene Ontology analyses detected 113 co-expressed unigenes associated with lipid biosynthesis. Of these, 15 showed significant changes in expression levels ( $\log _{2}$ fold values $\geq 1$ ) during seed development. Pathway analysis revealed 24 co-expressed transcripts involved in lipid biosynthesis and fatty acid biosynthesis pathways. These results provide a comprehensive molecular biology background for research on soybean seed development, particularly with respect to the process of oil accumulation. All of the genes identified in our research have significance for breeding soybeans with increased oil contents.

\section{Additional files} Additional file 1: Table S1. Differentially expressed genes between 35
DAF and 15 DAF (FDR $\leq 0.001$ and $\mid \log _{2}$ ratio| $\geq 1$ ).

Additional file 2: Table S2. Differentially expressed genes between 55 DAF and 15 DAF (FDR $\leq 0.001$ and $\mid \log _{2}$ ratio $\mid \geq 1$ ).

Additional file 3: Table S3. Differentially expressed genes between 65 DAF and 15 DAF (FDR $\leq 0.001$ and $\mid \log _{2}$ ratio| $\geq 1$ ).

Additional file 4: Table S4. Details of primers used for $\mathrm{QRT}$-PCR.

Additional file 5: Figure S1. Scatter plot of differentially expressed genes in seeds harvested at $35 \mathrm{DAF}, 55 \mathrm{DAF}$, and $65 \mathrm{DAF}$, compared with 15 DAF. (a). Scatter plot of differentially expressed genes between 35 DAF and 15 DAF. (b). Scatter plot of differentially expressed genes between 55 DAF and 15 DAF. (c). Scatter plot of differentially expressed genes between 65 DAF and 15 DAF. TPM = Transcript per million (normalized expression level of genes).

Additional file 6: Figure S2. Confirmation of differential gene expression by qRT-PCR.

\section{Abbreviations}

ACCase: acetyl CoA carboxylase ACP: acyl carrier protein BC: biotin carboxylase BCCP: biotin carboxyl carrier protein DAF: days after flowering DE: differential expression DEGs: differentially expressed genes

ER: endoplasmic reticulum FabA: 3-hydroxydecanoyl-[acyl-carrier-protein] dehydratase FabB, FabF, FabH or KASIII: 3-oxoacyl-[acyl-carrier-protein] synthase I, II, III: FabD or MCAT: [acyl-carrier-protein] S-malonyltransferase FabG: 3-oxoacyl-[acyl-carrier protein] reductase Fabl, FabK, FabL: enoyl-[acylcarrier protein] reductase I, II, III: FabZ: 3R-hydroxymyristoyl ACP dehydrase FASN, Fas: fatty acid synthase: FAS1, 2: fatty acid synthase subunit beta, alpha: FatA, FatB: fatty acyl-ACP thioesterase A, B: FDR: false discovery rate $\mathrm{MdcH}$ : malonate decarboxylase epsilon subunit RPKM: reads/Kb/million TAG: triacylglycerol TL: total lipids.

\section{Authors' contributions}

HC, FWW, YYD, NW, and YPS performed data analysis. HC, HYL wrote the manuscript. HYL and GC conceived the study. HC, HLY, YYJ, XYZ and YLL prepared the samples. HC, XYL, LL and XDF participated in the GRT-PCR verification. All the authors approved the final manuscript.

\section{Acknowledgments}

This work was supported by grants from the Program for the Special Program for Research of Transgenic Plants (Grant No. 2011ZX08010-002), the National Natural Science Foundation of China (Grant No. 30971804), the National High Technology Research and Development Program of China (863 Program) (Grant No. 2011AA100606), the New Century Excellent Talents in University (Grant No. NCET-08-0693), the Excellent innovation Team Project of Jilin Province, China (Grant No.20111815); the Science and Technology Development Project of Changchun City, Jilin, China (Grant No 2009024), and the State Key Laboratory of Crop Biology at Shandong Agricultural University, China (Grant No. 2010KF02).

Received: 11 December 2011 Accepted: 27 June 2012 Published: 31 July 2012

\section{References}

1. Zhou SQ, Zhang DJ, Luan HH, Yu FY, Xin XJ, Hu GH: Primary study on protein and lipid accumulation in high oil content soybean varieties. Chin. J. Oil Crop Sci 2006, 28(2):214-216.

2. Jako C, Kumar A, Wei YD, Zou JT, Barton DL, Giblin EM, Covello PS, Taylor DC: Seed-Specific Over-Expression of an Arabidopsis CDNA Encoding a Diacylglycerol Acyltransferase Enhances Seed Oil Content and Seed Weight ${ }^{1}$. Plant Physiol 2001, 126(2):861-874.

3. Wang HW, Zhang B, Hao YJ, Huang J, Tian AG, Liao Y, Zhang JS, Chen SY: The soybean Dof-type transcription factor genes, GmDof4 and GmDof11, enhance lipid content in the seeds of transgenic Arabidopsis plants. The Plant Journal 2007, 52(4):716-729.

4. Severin AJ, Woody JL, Bolon YT, Joseph B, Diers BW, Farmer AD, Muehlbauer GJ, Nelson RT, Grant D, Specht JE, Graham MA, Cannon SB, May GD, Vance CP, 
Shoemaker RC: RNA-Seq Atlas of Glycine max: A guide to the soybean transcriptome. BMC Plant Biol 2010, 10:160.

5. Levin JZ, Berger MF, Adiconis X, Rogov P, Melnikov A, Fennell T, Nusbaum C, Garraway LA, Gnirke A: Targeted next-generation sequencing of a cancer transcriptome enhances detection of sequence variants and novel fusion transcripts. Genome Biol 2009, 10(10):R115.

6. Zhang GJ, Guo GW, Hu XD, Zhang Y, Li QY, Li RQ, Zhuang RH, Lu ZK, He ZQ, Fang XD, Chen L, Tian W, Tao Y, Kristiansen K, Zhang XQ, Li SG, Yang HM, Wang J, Wang J: Deep RNA sequencing at single base-pair resolution reveals high complexity of the rice transcriptome. Genome Res 2010, 20(5):646-654

7. Jones SI, Gonzalez DO, Vodkin LO: Flux of transcript patterns during soybean seed development. BMC Genomics 2010, 11:136.

8. Dong XL, Bai PL, Wang JM, Ruan CJ: Comparative study on determination of seed oil content of energy plants by using NMR and Soxhlet Extraction. Renewable Energy Resources 2011, 29(3):21-24.

9. Mortazavi A, Williams BA, McCue K, Schaeffer L, Wold B: Mapping and quantifying mammalian transcriptomes by RNA-Seq. Nat Methods 2008, 5(7):621-628.

10. Nagalakshmi U, Wang Z, Waern K, Shou C, Raha D, Gerstein M, Snyder M: The Transcriptional Landscape of the Yeast Genome Defined by RNA Sequencing. Science 2008, 320(5881):1344-1349.

11. Hu RB, Fan CM, Li HY, Zhang QZ, Fu YF: Evaluation of putative reference genes for gene expression normalization in soybean by quantitative real-time RT-PCR. BMC Molecular Biology 2009, 10:93.

12. Yu J, Hu SN, Wang J, Wong GKS, Li SG, Liu B, Deng YJ, Dai L, Zhou Y, Zhang $X Q$, Cao ML, Liu J, Sun JD, Tang JB, Chen YJ, Huang XB, Lin W, Ye C, Tong W, Cong LJ, Geng JN, Han YJ, Li L, Li W, Hu GQ, Huang XG, Li WJ, Li J, Liu ZW, Li L, et al: A Draft Sequence of the Rice Genome (Oryza sativa L. ssp. indica). Science 2002, 296(5565):79-92.

13. Yu J, Wang J, Lin W, Li SG, Li H, Zhou J, Ni PX, Dong W, Hu SN, Zeng CQ, Zhang JG, Zhang Y, Li RQ, XU ZY, Li ST, Li XR, Zheng HK, Cong LJ, Lin L, Yin JN, Geng JN, Li GY, Shi JP, Liu J, Lv H, Li J, Wang J, Deng YJ, Ran LH, Shi XL, et al: The Genomes of Oryza sativa: A History of Duplications. PLoS Biol 2005, 3(2):e38.

14. Xia QY, Zhou ZY, Lu C, Cheng DJ, Dai FY, Li B, Zhao P, Zha XF, Cheng TC, Chai CL, Pan GQ, Xu JS, Liu C, Lin Y, Qian JF, Hou Y, Wu ZL, Li GR, Pan MH, Li CF, Shen YH, Lan XQ, Yuan LW, Li T, Xu HF, Yang GW, Wan YJ, Zhu Y, Yu $M D$, Shen WD, et al: A Draft Sequence for the Genome of the Domesticated Silkworm (Bombyx mori). Science 2004, 306(5703):1937-1940.

15. Sasaki Y, Nagano Y: Plant acetyl-CoA carboxylase: structure, biosynthesis, regulation, and gene manipulation for plant breeding. Biosci Biotechnol Biochem 2004, 68(6):1175-1184

16. Roesler K, Shintani D, Savage L, Boddupalli S, Ohlrogge J: Targeting of the Arabidopsis homomeric acetyl-coenzyme A carboxylase to plastids of rapeseeds. Plant Physiol 1997, 113(1):75-81.

17. Shintani D, Roesler K, Shorrosh B, Savage L, Ohlrogge J: Antisense expression and overexpression of biotin carboxylase in tobacco leaves. Plant Physiol 1997, 114(3):881-886.

18. Thelen JJ, Mekhedov S, Ohlrogge JB: Brassicaceae express multiple isoforms of biotin carboxyl carrier protein in a tissue-specific manner. Plant Physiol 2001, 125(4):2016-2028.

19. Elborough KM, Swinhoe R, Winz R, Kroon JT, Farnsworth L, Fawcett T, Martinez-Rivas JM, Slabas AR: Isolation of CDNAs from Brassica napus encoding the biotin-binding and transcarboxylase domains of acetylCoA carboxylase: assignment of the domain structure in a full-length Arabidopsis thaliana genomic clone. Biochem J 1994, 301(Pt 2):599-605.

20. Roesler KR, Shorrosh BS, Ohlrogge JB: Structure and expression of an Arabidopsis acetyl-coenzyme a carboxylase gene. Plant Physiol 1994, 105(2):611-617.

21. Simon JW, Slabas AR: cDNA cloning of Brassica napus malonyl-CoA: ACP transacylase (MCAT) (fabD) and complementation of an E. coli MCAT mutant. FEBS Lett 1998, 435(2-3):204-206.

22. Dehesh K, Tai H, Edwards P, Byrne J, Jaworski JG: Overexpression of 3-ketoacyl-acyl-carrier protein synthase Ills in plants reduces the rate of lipid synthesis. Plant Physiol 2001, 125(2):1103-1114.

23. Jaworski JG, Clough RC, Barnum SR: A cerulenin insensitive short chain 3-ketoacyl-acyl carrier protein synthase in Spinacia oleracea leaves. Plant Physiol 1989, 90(1):41-44.
24. Salas JJ, Ohlrogge JB: Characterization of substrate specificity of plant FatA and FatB acyl-ACP thioesterases. Arch Biochem Biophys 2002, 403(1):25-34.

25. Li-Beisson Y, Shorrosh B, Beisson F, Andersson MX, Arondel V, Bates PD, Baud S, Bird D, DeBono A, Durrett TP, Franke RB, Graham IA, Katayama K, Kelly AA, Larson T, Markham JE, Miquel M, Molina I, Nishida I, Rowland O, Samuels L, Schmid KM, Wada H, Welti R, Xu C, Zallot R, Ohlrogge J: Acyl-Lipid metabolism. The Arabidopsis Books 2010, 8:e0133.

26. Mandal MNA, Santha IM, Lodha ML, Mehta SL: Cloning of acyl-acyl carrier protein (ACP) thioesterase gene from Brassica juncea. Biochem Soc Trans 2000, 28(6):967-969

27. Dörmann $P$, Voelker $T A$, Ohlrogge JB: Accumulation of palmitate in Arabidopsis mediated by the acyl-acyl carrier protein thioesterase FATB $_{1}$. Plant Physiol 2000, 123(2):637-644.

28. Start WG, Ma Y, Polacco JC, Hildebrand DF, Freyer GA, Altschuler M: Two soybean seed lipoxygenase nulls accumulate reduced levels of lipoxygenase transcripts. Plant Mol Biol 1986, 7(1):11-23.

29. Craft DL, Madduri KM, Eshoo M, Wilson CR: Identification and Characterization of the CYP52 Family of Candida tropicalis ATCC 20336, Important for the Conversion of Fatty Acids and Alkanes to a, $\omega$-Dicarboxylic Acid. App Environ Micro 2003, 9(10):5983-5991.

\section{doi:10.1186/1471-2229-12-122}

Cite this article as: Chen et al.: Sequence mining and transcript profiling to explore differentially expressed genes associated with lipid biosynthesis during soybean seed development. BMC Plant Biology 2012 12:122.

\section{Submit your next manuscript to BioMed Central and take full advantage of:}

- Convenient online submission

- Thorough peer review

- No space constraints or color figure charges

- Immediate publication on acceptance

- Inclusion in PubMed, CAS, Scopus and Google Scholar

- Research which is freely available for redistribution 